\title{
Electrical Characterization of Nanopolyaniline/Porous Silicon Heterojunction at High Temperatures
}

\author{
Salah E. El-Zohary, ${ }^{1}$ M. A. Shenashen, ${ }^{1}$ Nageh K. Allam, ${ }^{2}$ T. Okamoto, ${ }^{1}$ and M. Haraguchi ${ }^{1}$ \\ ${ }^{1}$ Department of Optical Science and Technology, The University of Tokushima, 2-1 Minamijosanjima-cho, Tokushima, \\ Tokushima 770-8506, Japan \\ ${ }^{2}$ Energy Materials Laboratory, Physics Department, School of Sciences and Engineering, The American University in Cairo, \\ New Cairo 11835, Egypt \\ Correspondence should be addressed to Salah E. El-Zohary; elzohary@opt.tokushima-u.ac.jp
}

Received 28 June 2012; Revised 21 January 2013; Accepted 29 January 2013

Academic Editor: Xuedong Bai

Copyright (C) 2013 Salah E. El-Zohary et al. This is an open access article distributed under the Creative Commons Attribution License, which permits unrestricted use, distribution, and reproduction in any medium, provided the original work is properly cited.

\begin{abstract}
Nanopolyaniline/p-type porous silicon (NPANI/PSi) heterojunction films were chemically fabricated via in situ polymerization. The composition and morphology of the nanopolymer were confirmed using Fourier transform infrared, scanning electron microscopy, UV-visible, and transmission electron microscopy techniques. The results indicated that the polymerization took place throughout the porous layer. The $I-V$ measurements, performed at different temperatures, enabled the calculation of ideality factor, barrier height, and series resistance of those films. The obtained ideality factor showed a nonideal diode behavior. The series resistance was found to decrease with increasing temperature.
\end{abstract}

\section{Introduction}

Intrinsically conducting polymers, including polyacetylene, polyaniline (PANI), polypyrrole, polythiophene, poly(pphenylene-vinylene), and so forth, are termed organic polymers that possess the electrical, electronic, magnetic, and optical properties that are close to that of metals. Besides, they keep the same mechanical properties, processability, and so forth that are commonly associated with conventional polymers. Therefore, these polymers are commonly known as "synthetic metals" [1]. PANI is one of the most important classes of conjugated polymers that possess excellent electronic, optical, and redox properties. Also, making PANI with acids improves its environmental stability and conductivity $[2,3]$. PANI, as a conducting polymer, has been used in a plethora of practical applications such as battery electrodes $[4,5]$, electrochromic devices $[6,7]$, photoelectric cells $[8]$, light-emitting diodes $[9,10]$, solar cells $[11,12]$, energy storage [13], electromagnetic interference shielding [14], electrostatic discharge [15], biosensors [16, 17], and anticorrosive coatings $[18,19]$.
Various methods are available for the synthesis of PANI. However, the most widely used two techniques are electrochemical and chemical oxidative polymerization methods [20]. The chemical method is especially preferred as it can be used to produce PANI for large scale applications. Recently, there has been a great interest in the fabrication and assembly of nanostructured PANI, especially PANI nanofibers [21]. Since the first report by Huang et al. [22] that PANI nanofibers can be easily synthesized by an interfacial polymerization, many template-free methods have been established. For instance, PANI nanofibers were easily prepared by rapidly mixing acidic aqueous solutions of aniline and ammonium peroxydisulfate (APS) [23]. Also, nano-PANIs have been synthesized using dodecylbenzylsulfonic acid, which was successfully electrodeposited on the surface of glassy carbon electrodes to form nanostructured films suitable for heterogeneous catalysis applications [24].

As another class of important materials, porous silicon (PSi) is commonly prepared by chemical or electrochemical 
dissolution of crystalline silicon in HF-based solutions. Electrochemically fabricated PSi has a set of unique properties, especially the open porous structure and the enormous surface area. Moreover, PSi is much more elastic and pliant than the starting $\mathrm{Si}$ and thus can be used as a buffer between Si substrates and growing epitaxial films [25, 26].

Recently, there has been a great interest in the fabrication and assembly of organic/inorganic semiconductor heterostructures, which have been found promising in many applications, opening the door to new developments. For example, heterojunctions made of a thin layer of a molecular semiconductor deposited on the surface of an inorganic semiconductor substrate, such as $\mathrm{Si}, \mathrm{GaAs}$, or InP, have been found to form rectifiers with characteristics similar to ideal p-n junctions $[27,28]$. Of special interest, PANI/Si heterojunction has recently been studied thoroughly. Wang and Schiff [29] fabricated heterojunction solar cells using PANI on crystalline Si. They found the open-circuit voltage to increase with increasing the conductivity of the PANI film, saturating at a value of $0.51 \mathrm{~V}$. Dirani et al. [30] fabricated heterojunction diodes using poly(o-methoxyaniline)/PANI and amorphous/microcrystalline Si structures. They showed a dependence on the polymer doping level. Regarding the use of PSi, Chiboub et al. successfully prepared a PANI/PSi hybrid structure by oxidative chemical/electrochemical polymerization of an aniline-terminated PSi surface in the presence of aniline monomer in the solution. The results suggested that the PANI was covalently grafted onto the PSi surface, and that the polymerization took place throughout the porous layer. The resulting PANI/PSi hybrid interfaces showed a high chemical stability and good electrical properties [31]. Betty [32] presented an easy and inexpensive method for the fabrication of highly sensitive and specific immunosensor based on PANI/PS heterojunction. The PANI/PSi sensor was able to detect analyte-antibody interactions, while being reusable. A heterojunction between PSi and water-soluble copolymer of PANI was fabricated by Fan et al. [33], which showed rectifying characteristics that were enhanced with decreasing the degree of sulfonation and the thickness of the copolymer.

As the operating temperature of the devices made of such heterojunctions is a determinant factor of their efficiency, Aydoğan et al. [34] studied the $I-V$ characteristics of $\mathrm{Au} / \mathrm{PANI} / \mathrm{PSi} / \mathrm{Al}$ heterostructure in a wide range of temperatures $(90-300 \mathrm{~K})$. They showed a decrease in the barrier height $(\mathrm{BH})$ and an increase in the ideality factor (n) with decreasing temperature. The refractive index shows an anomalous dispersion in the absorption region and a normal behavior in the transparent region. Therefore, more studies are needed to evaluate the performance of such heterojunctions at different temperatures.

Herein, we demonstrate the in situ chemical fabrication of nano-PANI/PSi heterojunction films and their characterization using FTIR, UV-visible, X-Ray, SEM, and TEM techniques. The $I-V$ characteristics of the films are studied at different temperatures (298-398 K) and discussed based on the thermionic model. The electronic parameters (e.g., $n$, $\mathrm{BH}$, and resistance) of the fabricated heterostructures are also determined.
1.1. Materials. Aniline (Riedel-de Haën, puriss grade) was distilled twice under atmospheric pressure and over zinc dust before use. Ammonium peroxydisulfate (APS) (WIN$\mathrm{LAB}, \mathrm{UK}$ ) and cetyltrimethylammonium bromide (CTAB) (Fluka) were used without further purification. Sulfuric acid $\left(\mathrm{H}_{2} \mathrm{SO}_{4}\right) 96 \%$, hydrogen peroxide $\left(\mathrm{H}_{2} \mathrm{O}_{2}\right) 30 \%$ (SigmaAldrich), hydrofluoric acid (HF) 47-51\% (Fluka), and ethanol and methanol were used as received.

1.2. Preparation of PSi Substrates. Double-side-polished $\mathrm{p}$ type $\mathrm{Si}(100)$ wafer (boron-doped, $5-10 \Omega \mathrm{cm}$ resistivity) was first cleaned in $3: 1$ concentrated $\mathrm{H}_{2} \mathrm{SO}_{4} / \mathrm{H}_{2} \mathrm{O}_{2}$ for $5 \mathrm{~min}$ at $80^{\circ} \mathrm{C}$ and then sonicated in deionized water for $10 \mathrm{~min}$. Consequently, the wafer was rinsed in acetone to remove the cleaning residue and acts as a further cleaning solvent, after that the wafer was immersed in methanol for $3 \mathrm{~min}$ to remove any acetone residue. Finally, the wafer was rinsed in deionized water for $3 \mathrm{~min}$ to remove methanol residue. The clean wafers were immersed in $\mathrm{HF}: \mathrm{H}_{2} \mathrm{O}(1: 10)$ solution for $20 \mathrm{~s}$ at room temperature to remove the native oxide. The hydrogen-terminated surfaces were electrochemically etched in a $1: 1: 3$ (by volume) solution of $\mathrm{HF}$ : ethanol: $\mathrm{H}_{2} \mathrm{O}$ for $15 \mathrm{~min}$. After etching, the samples were rinsed with ethanol and deionized water and then dried under a stream of dry nitrogen prior to use.

1.3. Preparation of PANI Nanoparticles/PSi Heterojunction. CTAB $(0.05 \mathrm{~g}, 0.69 \mathrm{mM})$ was dissolved in doubly deionized water $(40 \mathrm{~mL})$ and stirred at room temperature. Aniline $(0.05 \mathrm{M})$ was added to the CTAB solution and stirred for an additional one hour at room temperature $\left(15 \pm 1^{\circ} \mathrm{C}\right)$. APS solution $(0.0625 \mathrm{M})$ dissolved in deionized water $(40 \mathrm{~mL})$ was added to aniline solution at room temperature. Five $\mathrm{mL}$ of this mixture was then taken and poured carefully on the surface of PSi wafer. The remaining solution $(75 \mathrm{~mL})$ was left to complete the reaction. The originally colorless mixture turned blue and later became dark green. The resultant green color content was allowed to stand for $15 \mathrm{~h}$ without being disturbed. The solid mass was filtrated and washed with distilled water and methanol several times until the filtrate became colorless. The solid product was then dried in a vacuum oven at $60^{\circ} \mathrm{C}$ for $48 \mathrm{~h}$ to be ready for analytical measurements. Also, the wafer was washed with deionized water several times.

\section{Characterization}

2.1. UV-Visible Spectra. PANI films were obtained during the polymerization of aniline on rectangular glass slides. These slides were introduced into the reaction vessel before the polymerization starts. Once the reactants were introduced to the vessel, the film grows onto the glass support simultaneously. After time intervals during the polymerization process, the glass supports were removed from the reaction mixture and rinsed with deionized water and finally dried. These supports coated with PANI films were used for the UV-visible absorption measurements. The absorption spectra were recorded with a Shimadzu UV-2101 DC spectrophotometer. Uncoated glass supports were used as references. 

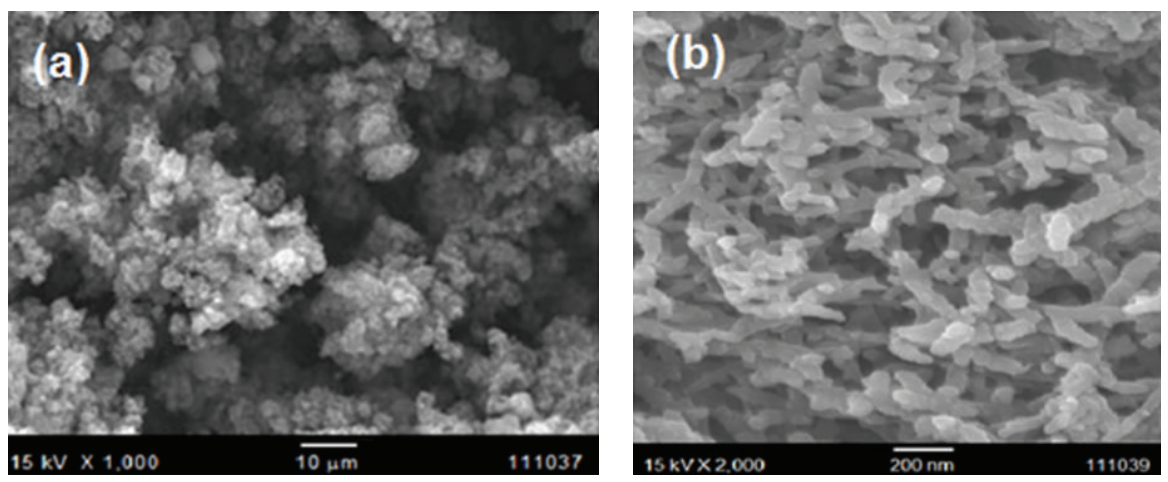

FIGURE 1: NPANI SEM micrographs and rod-like-shaped nanostructures.

2.2. FTIR Measurements. FTIR spectra of the PANI samples dispersed in a $\mathrm{KBr}$ pellet were obtained using a JASCO 410 instrument.

2.3. Scanning Electron Microscope (SEM). A morphological study was performed via SEM observation of the cross section of PANI nanoparticles using a SEM (model JOEL14775) at $10 \mathrm{kV}$ with gold coating on the samples.

2.4. Transmission Electron Microscopic Analysis (TEM). The nanostructure of deposited PANI film was confirmed by TEM technique. TEM measurements were taken using a JEOL JEM-2000EX microscope at an accelerating voltage of $100 \mathrm{kV}$. For TEM observation, the PANI nanoparticles were placed onto a carbon film supported by a copper grid.

2.5. I-V Measurements. Current-voltage $(I-V)$ characteristics of the heterojunction films were studied at different temperatures (298-398 K) using Keithley 2400 source meter. The temperature was continuously monitored by using thermocouple close to the sample.

\section{Result and Discussion}

3.1. Morphological, Structural, and Optical Properties. SEM was used to investigate the surface morphology of the NPANI synthesized in CTAB micelles. In the SEM micrographs (Figures 1(a) and 1(b)), rod-like shape nanostructures can be distinguished (Figure 1(b)) with diameters ranging from 30-50 nm, which is in good agreement with the TEM results shown in Figure 2. The obtained diameters are much smaller with very narrow size distribution compared to those reported by Guo and Zhou who synthesized PANI nanofibers with diameters in the range $80-100 \mathrm{~nm}$ by electrochemical polymerization [35] and electrospun PANI with diameters of $20-150 \mathrm{~nm}$ [36]. However, these are larger than PANI nanofibers synthesized using aluminum oxide template (20$40 \mathrm{~nm}$ in diameter) [37] and $\mathrm{FeCl}_{3}$ as an oxidant (16-23 nm in diameter) [38]. In our study, the micelles formed by CTAB and aniline might act as templates for the formation of the obtained self-assembled PANI nanorods. Upon the addition of APS, polymerization takes place only at the micelle/water interface. In addition, as PANI is a rigid molecule, the micelles have a tendency to elongate in the direction of the polymer chain during the polymerization process resulting in the observed PANI nanorods.

Figure 3 represents the FTIR spectrum of the fabricated NPANI film. The characteristic sharp band that appeared at $1220-1020 \mathrm{~cm}^{-1}$ can be assigned to the $\mathrm{C}-\mathrm{N}$ tertiary aromatic vibration, while the bands located at 1360, 1250, 1340, and $1310 \mathrm{~cm}^{-1}$ are due to the $\mathrm{C}-\mathrm{N}$ primary and secondary vibrations. The sharp characteristic band at $3450-3200 \mathrm{~cm}^{-1}$ is due to the N-H stretching vibration. Moreover, the bands at 1550 and $1475 \mathrm{~cm}^{-1}$ are due to the presence of quinoid structure, and the band obtained at $1240 \mathrm{~cm}^{-1}$ can be related to the $\mathrm{C}-\mathrm{N}$ stretching mode for the benzenoid ring. The band at $810 \mathrm{~cm}^{-1}$ is a characteristic of a p-substituted aromatic ring proposing that polymerization has proceeded in a "head to tail" form.

For more justification, Figure 4 shows the UV-visible spectrum of NPANI film deposited on a glass substrate. It can be shown that the spectrum shows two bands; one at $345 \mathrm{~nm}$ corresponds to the $\pi-\pi^{*}$ transition of the benzenoid rings, and another broad band at $795 \mathrm{~nm}$ corresponds to the quinine structure.

3.2. I-V Measurements. According to the thermionic emission model, for a metal/semiconductor $(\mathrm{M} / \mathrm{S})$ or metal/polymer $(\mathrm{M} / \mathrm{P})$ contact [39-42], the $I-V$ characteristic under the forward and reverse bias voltages through an SBD can be represented as

$$
I=I_{0}\left(\frac{q V}{\eta K T}\right)\left[1-\exp \left(\frac{q V}{K T}\right)\right]
$$

where $I$ is the forward diode current, $I_{0}$ is the reverse saturation current, $q$ is the electron charge, $V$ is the applied bias voltage, $k$ is the Boltzmann's constant, $T$ is the absolute temperature, and $\eta$ is the ideality factor. The reverse saturation current $\left(I_{0}\right)$ can be obtained by extrapolating the linear portion of the forward bias in the $\ln I-V$ plot to the intercept point on the current axis at zero-bias $(V=0)$ and is given by

$$
I_{0}=A A^{*} T^{2} \exp \left(-\frac{q \Phi_{B}}{k T}\right)
$$




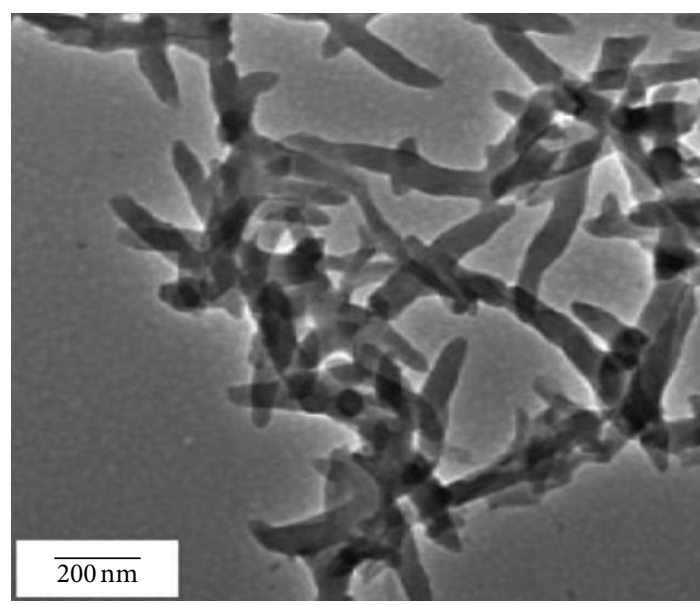

FIgURE 2: A TEM micrograph of synthesized rod-like NPANI.

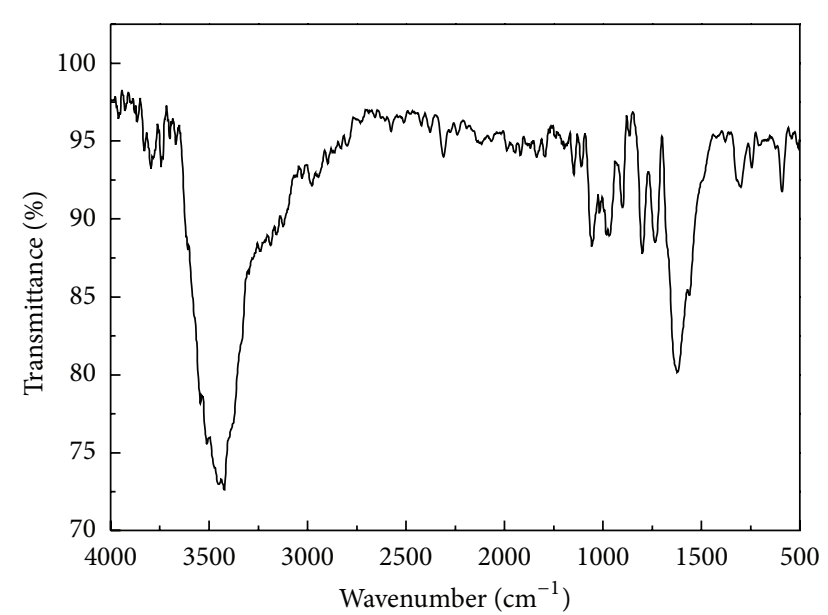

FIGURE 3: FTIR spectrum of the synthesized NPANI.

where $A$ is the effective diode area, $A^{*}$ is the effective Richardson constant (32 A/ $\mathrm{cm}^{2} \mathrm{~K}^{2}$ for p-type Si [43]), and $\Phi_{B}$ is the zero-bias barrier height.

The ideality factor $(\eta)$, a dimensionless quantity, is a measure of the conformity of the diode behavior to pure thermionic emission. It is introduced to account for the deviation from the thermionic emission diffusion theory. $\eta$ can be determined from the slope of the straight line region of the ln $I-V$ plot and can be represented as

$$
\eta=\frac{q}{k T}\left(\frac{d V}{d(\ln I)}\right)
$$

For an ideal diode, $\eta$ is unity. However, values greater than unity are usually observed and attributed to many factors such as the presence of interfacial thin native oxide layer, series resistance, and so forth [44]. Also, barrier inhomogeneity has been used to explain the higher values of the ideality factor [9]. For the estimation of the barrier height $\left(\Phi_{B}\right)$,

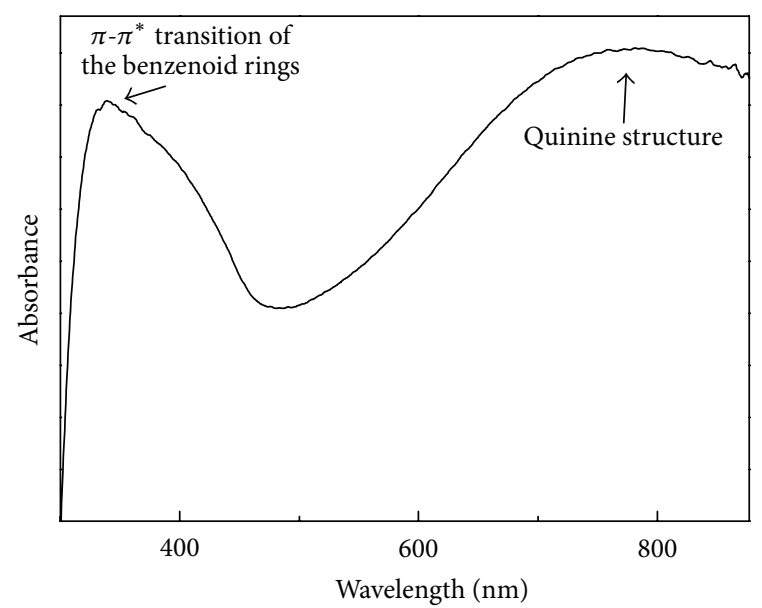

FIGURE 4: UV-visible spectrum of the synthesized NPANI.

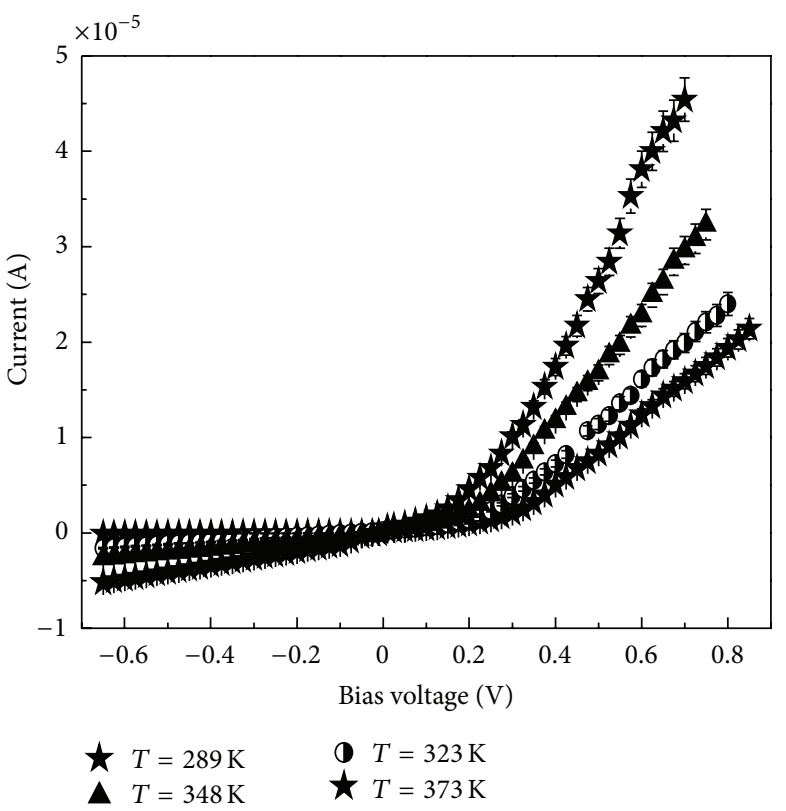

FIGURE 5: $I-V$ characteristics of NPANI/PSi heterojunction at different temperatures.

one may also make use of the Richardson plot of saturation current, that is,

$$
\ln \left(\frac{I_{0}}{T^{2}}\right)=\ln \left(A A^{*}\right)-\frac{q \Phi_{B o}}{K T} .
$$

Based on the above analysis, the $I-V$ measurements were done for the NPANI/PSi films to determine the transport mechanism in such diodes. Figure 5 shows the $I-V$ characteristics under the forward and reverse bias conditions at different temperatures in the range $298-373 \mathrm{~K}$. The curves are similar to that of a metal/semiconductor (MS) Schottky barrier (SB). Asymmetrical $I-V$ characteristic under the forward and reverse bias voltages is observed. In the low voltages range, the forward current increases with increasing temperature indicating a negative resistance temperature 


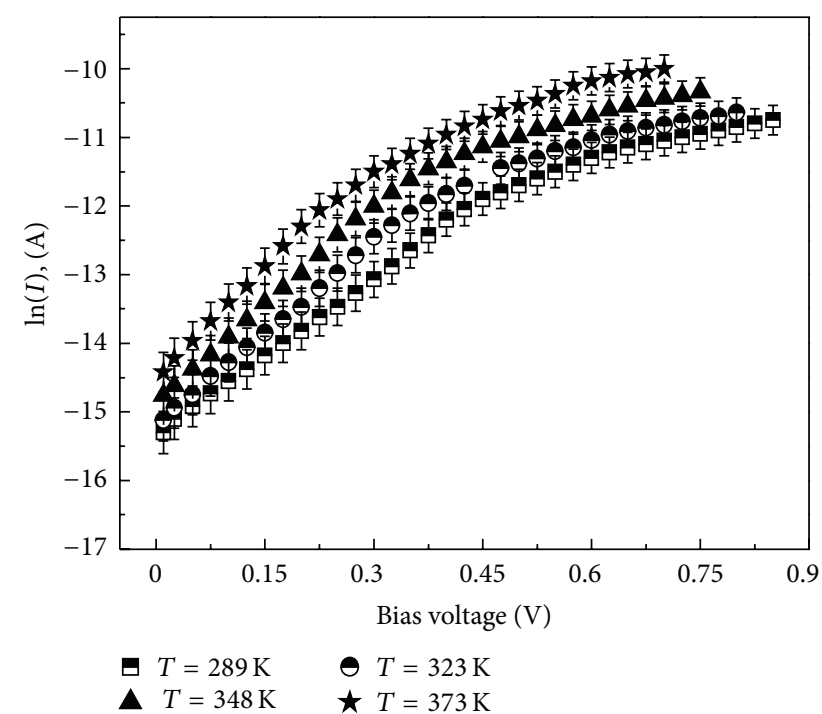

FIGURE 6: $\ln (I)$ vis bias voltage (V) for NPANI/PSi heterojunction at various temperatures in dark.

coefficient. The observed exponential dependence may be due to the formation of a depletion region between NPANI layer and PSi film.

It is observed that the junction exhibits strong rectifying characteristics in dark showing an $\mathrm{n}$-p heterojunction as diode behavior. This behavior can be attributed to the barrier at the interface that limits the forward and reverse carriers flow across the junction, where the built-in potential could be developed [39]. The forward $I-V$ characteristics of NPANI/PSi SB diode, according to thermionic emission theory [40], are expressed by (1). As can be seen in Figure 5, the forward bias of the structure current is an exponential function of the applied bias voltage in the intermediate voltage regime $(V \geq 0.35 \mathrm{~V})$.

To confirm that the thermionic emission is the operating conduction mechanism, the experimental values of $\eta$ and $\Phi_{B}$ at each temperature were determined using (3) and (4), respectively. $\eta$ is estimated from the slope of the linear region of the forward bias plot shown in Figure 6. The obtained $\eta$ values (2.8-5.45) indicate that the NPANI/PSi SBD represents a nonideal diode. Moreover, the obtained values $(\eta>1)$ can be generally attributed to the presence of a bias-dependent Schottky barrier height "SBH.'

For an inhomogeneous SBD, with a distribution of low $\mathrm{SBH}, \eta$ may increase with decreasing temperature [44]. The SB consists of laterally inhomogeneous patches of different barrier heights. Since current transport across the organic/inorganic interface is a temperature-activated process, at low temperature electrons are able to surmount the lower barriers and, therefore, the current transport will be dominated by the current flowing through the patches of lower SBH and larger $\eta[39,45,46]$. The high values of $\eta$ are also probably due to a potential drop in the interface layer and presence of excess current as well as the recombination current through the interfacial states between the PSi substrate and the NPANI organic layer $[44,45]$. In this case, $\Phi_{B}$ is the

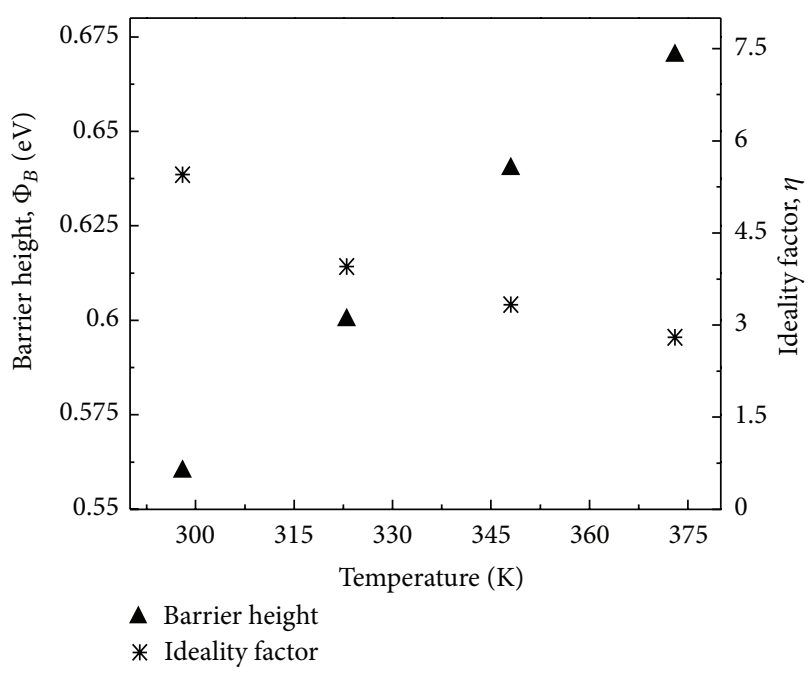

FIGURE 7: Temperature dependence of barrier height and ideality factor.

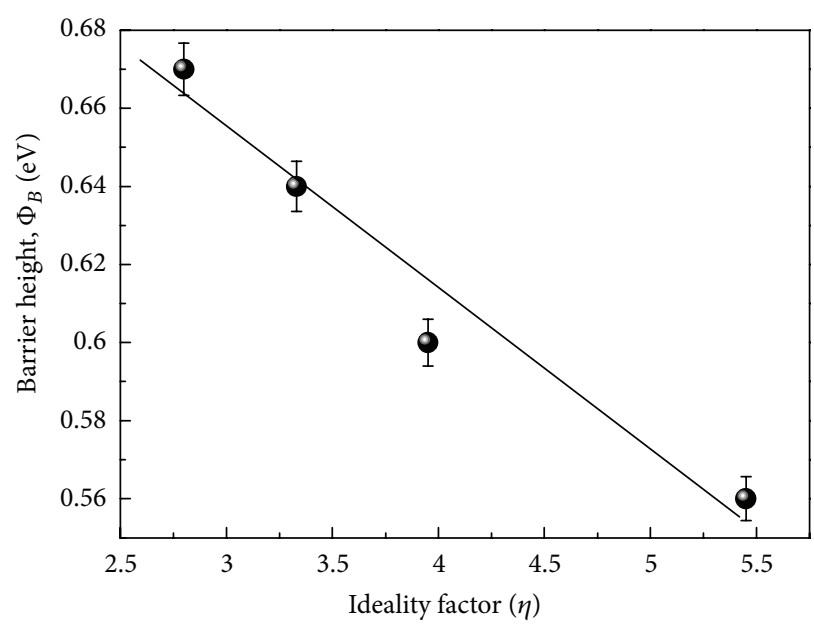

FIGURE 8: Barrier height versus ideality factor for the NPANI/PSi heterojunction.

contact potential barrier that exists at the interface between organic material and semiconductor layer.

Using linear curve fitting of Figure $6, \Phi_{B}$ was found to increase with increasing temperature, Figure 7 , while $\eta$ is decreasing with increasing temperature, in agreement with previous reports $[34,39]$. It is important to note that such temperature dependence is an obvious disagreement with the reported negative temperature coefficient of SBHs.

Upon increasing the temperature, more and more electrons will have sufficient energy to surmount the higher barrier. As a result, the dominant $\Phi_{B}$ will increase with increasing temperature and bias voltage [40]. Therefore, the current flow through the lower $\Phi_{B}$ and larger $\eta$ will dominate the current transport mechanism

Figure 8 shows the variation of $\Phi_{B}$ versus $\eta$ for the tested PANI/PSi heterojunction. Note that a linear relationship between $\Phi_{B}$ and $\eta$ is obtained. This can be an evidence that the current transport across the organic/inorganic interface 
TABLE 1: Saturation current $\left(I_{0}\right)$, barrier height $\left(\Phi_{B}\right)$, ideality factor $(\eta)$, and $R_{s}$ factor for NPANI/PSi heterojunction.

\begin{tabular}{lccccc}
\hline \multirow{2}{*}{$T(\mathrm{~K})$} & \multirow{2}{*}{$I_{0}(\mathrm{~A})$} & \multirow{2}{*}{$\Phi_{B}(\mathrm{eV})$} & \multicolumn{2}{c}{$d V / d(\ln I)$} \\
& & & & $\eta$ & $R_{s}(\mathrm{k} \Omega)$ \\
\hline 298 & $8.52782 \times 10^{-8}$ & 5.45 & 0.56 & 4.47 & 28.8 \\
323 & $1.17942 \times 10^{-7}$ & 3.95 & 0.60 & 3.34 & 16.8 \\
348 & $1.83184 \times 10^{-7}$ & 3.33 & 0.64 & 2.78 & 10.2 \\
373 & $2.91448 \times 10^{-7}$ & 2.80 & 0.67 & 2.24 & 5.81 \\
\hline
\end{tabular}

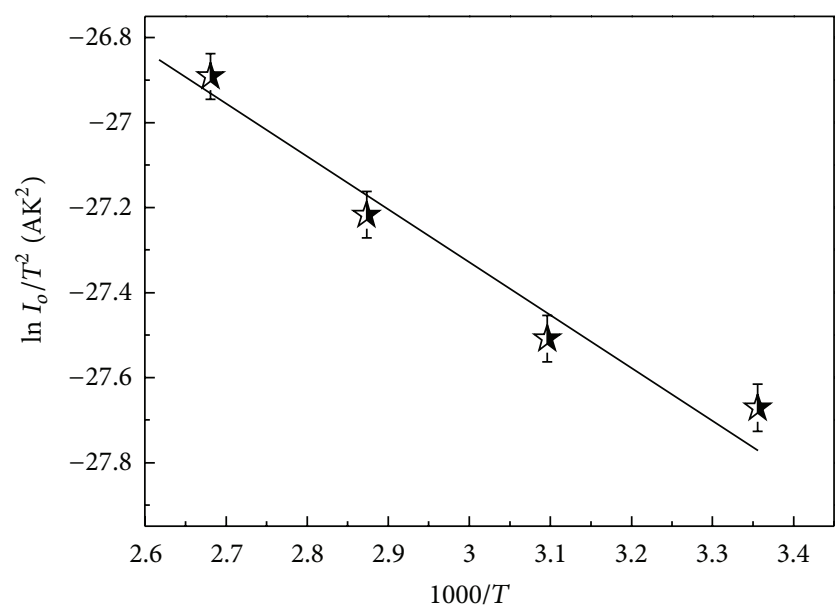

FIGURE 9: Richardson plot for the NPANI/PSi heterojunction.

is a temperature-dependent activated process, which can be explained by lateral inhomogeneities of the barrier heights in the diodes [45].

For the evaluation of $\Phi_{B}$, one may also make use of the Richardson plot of saturation current. Figure 9 shows the variation of $\ln \left(I_{0} / T^{2}\right)$ versus $1000 / T$ where linear relation was observed over the entire range of temperature. This indicates that thermionic emission is the dominant mechanism of charge transport. A Richardson constant $\left(A^{*}\right)$ value of $2.8297 \times 10^{-4} \mathrm{AK}^{-2} \mathrm{~cm}^{-2}$ is determined from intercept at the ordinate of this experimental plot, which is much lower than the corresponding reported value $\left(32 \mathrm{AK}^{-2} \mathrm{~cm}^{-2}\right)$ for $\mathrm{p}-\mathrm{Si}$. This deviation may be due to the spatial inhomogeneous barrier heights and potential fluctuations at the interface that consist of low and high barrier areas [41, 42].

According to the thermionic emission theory, the forward bias $I-V$ characteristics of a Schottky diode with a series resistance can be approximately expressed as

$$
I=I_{0} \exp \left[\frac{q\left(V-I R_{s}\right)}{\eta k T}\right],
$$

where the $I R_{s}$ is the voltage drop across the series resistance $\left(R_{s}\right)$ of the device. The series resistance can be determined using

$$
\frac{d V}{d(\ln I)}=\frac{\eta k T}{q}+I R_{s}
$$

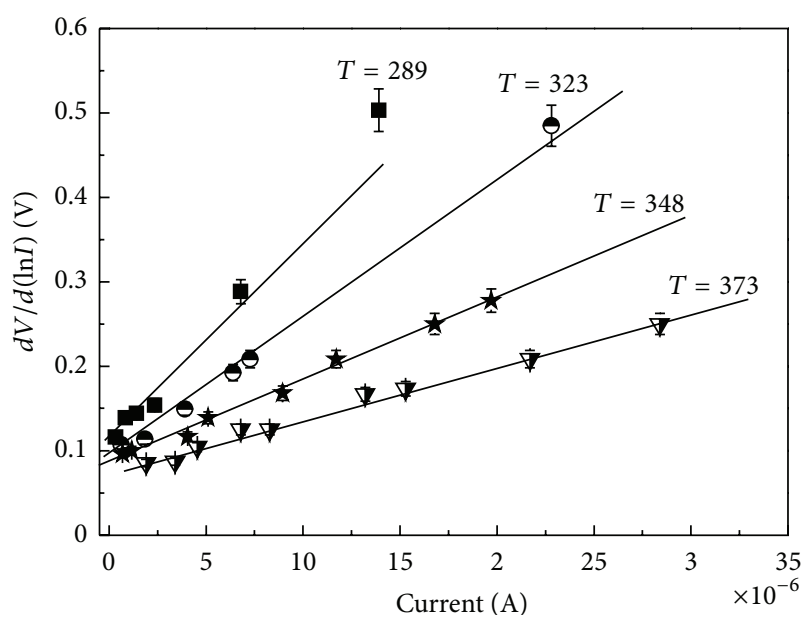

FIGURE 10: $d V / d(\ln I)-I$ curves of the NPANI/PSi heterojunction at different temperatures.

The values of saturation current $\left(I_{0}\right)$, barrier height $\left(\Phi_{B}\right)$, ideality factor $(\eta)$, and $R_{s}$ factor for NPANI/PSi heterojunction were calculated from the forward $I-V$ characteristics at different temperatures and listed in Table 1.

A plot of $(d V / d \ln I)$ versus $(I)$ is linear with the slope giving the $R_{s}$, and $\eta$ can be estimated from the intercept $(\eta k T / q)$. Temperature dependence of the obtained $R_{s}$ is shown in Figure 10 . The $R_{s}$ values vary exponentially with temperature in the temperature range $298-398 \mathrm{~K}$. The decrease in $R_{s}$ with increasing temperature can be attributed to the factors responsible for increasing $\eta$ and/or to the lack of free carriers at low temperatures [47].

\section{Conclusion}

The morphological, structural, and optical properties of the fabricated nanopolyaniline (NPANI) were characterized by SEM, TEM, FTIR, and UV-visible techniques. Different parameters for the assembled NPANI/PSi heterojunction were calculated from $I-V$ measurements performed in the dark conditions in the temperature range $298-373 \mathrm{~K}$. It has been found that while the zero-bias barrier height decreases, the ideality factor increases with decreasing temperature. This behavior has been attributed to particular distribution of barrier heights, barrier inhomogeneity, and interface states localized at the NPANI/PSi SBD. The calculated ideality factor (2.8-5.45) indicates that NPANI/PSi SBD represents a nonideal diode. The series resistance values are decreased with increasing temperature, which was attributed to the factors responsible for the increase in $\eta$ and/or to the lack of free carriers at low temperatures. The results suggested that the NPANI was covalently grafted onto the PSi surface, and polymerization took place throughout the porous layer.

\section{Acknowledgment}

The authors would like to acknowledge the financial support received from NEC C\&C Foundation, Japan. 


\section{References}

[1] A. G. MacDiarmid, "Synthetic metals: a novel role for organic polymers," Synthetic Metals, vol. 125, no. 1, pp. 11-22, 2002.

[2] E. T. Kang, K. G. Neoh, and K. L. Tan, "Polyaniline: a polymer with many interesting intrinsic redox states," Progress in Polymer Science, vol. 23, no. 2, pp. 277-324, 1998.

[3] H. Namazi, R. Kabiri, and A. Entezami, "Determination of extremely low percolation threshold electroactivity of the blend polyvinyl chloride/polyaniline doped with camphorsulfonic acid by cyclic voltammetry method," European Polymer Journal, vol. 38, no. 4, pp. 771-777, 2002.

[4] A. G. MacDiarmid, S.-L. Mu, N. L. D. Somasiri, and W. Wu, "Electrochemical characteristics of "polyaniline" cathodes and anodes in aqueous electrolytes," Molecular Crystals and Liquid Crystals, vol. 121, pp. 187-190, 1985.

[5] P. Novák, K. Müller, K. S. Santhanam, and O. Haas, "Electrochemically active polymers for rechargeable batteries," Chemical Reviews, vol. 97, no. 1, pp. 207-282, 1997.

[6] C. D. Batich, H. A. Laitinen, and H. C. Zhou, "Chromatic changes in polyaniline films," Journal of the Electrochemical Society, vol. 137, no. 3, pp. 883-885, 1990.

[7] S. A. Sapp, G. A. Sotzing, and J. R. Reynolds, "High contrast ratio and fast-switching dual polymer electrochromic devices," Chemistry of Materials, vol. 10, no. 8, pp. 2101-2108, 1998.

[8] Y. H. Dong and S. L. Mu, "Photoelectrochemical behaviour of polyaniline affected by potentials and $\mathrm{pH}$ of solutions," Electrochimica Acta, vol. 36, no. 13, pp. 2015-2018, 1991.

[9] J. Jang, J. Ha, and K. Kim, "Organic light-emitting diode with polyaniline-poly(styrene sulfonate) as a hole injection layer," Thin Solid Films, vol. 516, no. 10, pp. 3152-3156, 2008.

[10] S. A. Chen, K. R. Chuang, C. I. Chao, and H. T. Lee, "White-light emission from electroluminescence diode with polyaniline as the emitting layer," Synthetic Metals, vol. 82, no. 3, pp. 207-210, 1996.

[11] Q. Li, J. Wu, Q. Tang et al., "Application of microporous polyaniline counter electrode for dye-sensitized solar cells," Electrochemistry Communications, vol. 10, no. 9, pp. 1299-1302, 2008.

[12] S. X. Tan, J. Zhai, M. X. Wan, L. Jiang, and D. B. Zhu, "Polyaniline as a hole transport material to prepare solid solar cells," Synthetic Metals, vol. 137, no. 1-3, pp. 1511-1512, 2003.

[13] X. Zhang, W. J. Goux, and S. K. Manohar, "Synthesis of polyaniline nanofibers by "nanofiber seeding"', Journal of the American Chemical Society, vol. 126, no. 14, pp. 4502-4503, 2004.

[14] Y. Wang and X. Jing, "Intrinsically conducting polymers for electromagnetic interference shielding," Polymers for Advanced Technologies, vol. 16, no. 4, pp. 344-351, 2005.

[15] F. Wiznerowicz, "Conductive polymers instead of carbon black?” Wire, vol. 44, no. 1, p. 102, 1994.

[16] M. Gerard, A. Chaubey, and B. D. Malhotra, "Application of conducting polymers to biosensors," Biosensors and Bioelectronics, vol. 17, no. 5, pp. 345-359, 2002.

[17] R. N. Silva, E. R. Asquieri, and K. F. Fernandes, "Immobilization of Aspergillus niger glucoamylase onto a polyaniline polymer," Process Biochemistry, vol. 40, no. 3-4, pp. 1155-1159, 2005.

[18] D. E. Tallman, G. Spinks, A. Dominis, and G. G. Wallace, "Electroactive conducting polymers for corrosion control: part 1. General introduction and a review of non-ferrous metals," Journal of Solid State Electrochemistry, vol. 6, no. 2, pp. 73-84, 2002.
[19] A. Kalendová, D. Veselý, J. Stejskal, and M. Trchová, "Anticorrosion properties of inorganic pigments surface-modified with a polyaniline phosphate layer," Progress in Organic Coatings, vol. 63, no. 2, pp. 209-221, 2008.

[20] J. C. Chiang and A. G. MacDiarmid, "“Polyaniline”: protonic acid doping of the emeraldine form to the metallic regime," Synthetic Metals, vol. 13, no. 1-3, pp. 193-205, 1986.

[21] D. Zhang and Y. Y. Wang, "Synthesis and applications of one-dimensional nano-structured polyaniline: an overview," Materials Science and Engineering: B, vol. 134, pp. 9-19, 2006.

[22] J. Huang, S. Virji, B. H. Weiller, and R. B. Kaner, "Polyaniline nanofibers: facile synthesis and chemical sensors," Journal of the American Chemical Society, vol. 125, no. 2, pp. 314-315, 2003.

[23] J. X. Huang and R. B. Kaner, "Nanofiber formation in the chemical polymerization of aniline: a mechanistic study," Angewandte Chemie, vol. 116, no. 43, pp. 5941-5945, 2004.

[24] S. E. Moulton, P. C. Innis, L. A. P. Kane-Maguire, O. Ngamna, and G. G. Wallace, "Polymerisation and characterisation of conducting polyaniline nanoparticle dispersions," Current Applied Physics, vol. 4, no. 2-4, pp. 402-406, 2004.

[25] M. Mynbaeva, A. Sitnikova, A. Tregubova, and K. Mynbaev, "HVPE GaN growth on porous $\mathrm{SiC}$ with closed surface porosity," Journal of Crystal Growth, vol. 303, no. 2, pp. 472-479, 2007.

[26] A. A. M. Farag, A. Ashery, and F. S. Terra, "Fabrication and electrical characterization of $\mathrm{n}$-InSb on porous Si heterojunctions prepared by liquid phase epitaxy," Microelectronics Journal, vol. 39, no. 2, pp. 253-260, 2008.

[27] S. R. Forrest, M. L. Kaplan, and P. H. Schmidt, "Organicon-inorganic semiconductor contact barrier diodes. I. Theory with applications to organic thin films and prototype devices," Journal of Applied Physics, vol. 55, no. 6, pp. 1492-1507, 1984.

[28] P. Kumar, S. Adhikari, and P. Banerji, "Fabrication and characterization of polyaniline/porous silicon heterojunction," Synthetic Metals, vol. 160, no. 13-14, pp. 1507-1512, 2010.

[29] W. Wang and E. A. Schiff, "Polyaniline on crystalline silicon heterojunction solar cells," Applied Physics Letters, vol. 91, no. 13, Article ID 133504, 3 pages, 2007.

[30] E. A. T. Dirani, R. K. Onmori, C. A. Olivati, R. M. Faria, and A. M. Andrade, "Study of heterojunction diodes using POMA/PANI and amorphous/microcrystalline silicon structures," Synthetic Metals, vol. 121, no. 1-3, pp. 1545-1546, 2001.

[31] N. Chiboub, R. Boukherroub, N. Gabouze et al., "Covalent grafting of polyaniline onto aniline-terminated porous silicon," Optical Materials, vol. 32, no. 7, pp. 748-752, 2010.

[32] C. A. Betty, "Highly sensitive capacitive immunosensor based on porous silicon-polyaniline structure: bias dependence on specificity," Biosensors and Bioelectronics, vol. 25, no. 2, pp. 338343, 2009.

[33] J. Fan, M. Wan, and D. Zhu, "Studies on the rectifying effect of the heterojunction between porous silicon and water-soluble copolymer of polyaniline," Synthetic Metals, vol. 95, no. 2, pp. 119-124, 1998.

[34] Ş. Aydoğan, M. Sağlam, and A. Türüt, "On the barrier inhomogeneities of polyaniline/p-Si/Al structure at low temperature," Applied Surface Science, vol. 250, no. 1, pp. 43-49, 2005.

[35] Y. Guo and Y. Zhou, "Polyaniline nanofibers fabricated by electrochemical polymerization: a mechanistic study," European Polymer Journal, vol. 43, no. 6, pp. 2292-2297, 2007.

[36] N. J. Pinto, I. Ramos, R. Rojas, P. C. Wang, and A. T. Johnson, "Electric response of isolated electrospun polyaniline nanofibers to vapors of aliphatic alcohols," Sensors \& Actuators $B$, vol. 129, no. 2, pp. 621-627, 2008. 
[37] Z. Wang, M. Chen, and H. L. Li, "Preparation and characterization of uniform polyaniline nano-fibrils using the anodic aluminum oxide template," Materials Science and Engineering A, vol. 328, no. 1, pp. 33-38, 2002.

[38] Z. Zhang, J. Deng, and M. Wan, "Highly crystalline and thin polyaniline nanofibers oxidized by ferric chloride," Materials Chemistry and Physics, vol. 115, no. 1, pp. 275-279, 2009.

[39] R. T. Tung, "Electron transport of inhomogeneous Schottky barriers," Applied Physics Letters, vol. 58, no. 24, pp. 2821-2823, 1991.

[40] C. Coskun, M. Biber, and H. Efeoglu, "Temperature dependence of current-voltage characteristics of Sn/p-GaTe Schottky diodes," Applied Surface Science, vol. 211, no. 1-4, pp. 360-366, 2003.

[41] J. H. Werner and H. H. Güttler, "Barrier inhomogeneities at Schottky contacts," Journal of Applied Physics, vol. 69, no. 3, pp. 1522-1533, 1991.

[42] C. A. Dimitriadis, S. Logothetidis, and I. Alexandrou, "Schottky barrier contacts of titanium nitride on n-type silicon," Applied Physics Letters, p. 502, 1995.

[43] Ş. Aydoǧan, M. Sağlam, and A. Türüt, "Some electrical properties of polyaniline/p-Si/Al structure at $300 \mathrm{~K}$ and $77 \mathrm{~K}$ temperatures," Microelectronic Engineering, vol. 85, no. 2, pp. 278-283, 2008.

[44] M. Pattabi, S. Krishnan, Ganesh, and X. Mathew, "Effect of temperature and electron irradiation on the I-V characteristics of Au/CdTe Schottky diodes," Solar Energy, vol. 81, no. 1, pp. 111116, 2007.

[45] D. T. Quan and H. Hbib, "High barrier height Au/n-type InP Schottky contacts with a POxNyHz interfacial layer," Solid-State Electronics, vol. 36, no. 3, pp. 339-344, 1993.

[46] A. Gümüş, A. Türüt, and N. Yalçin, “Temperature dependent barrier characteristics of CrNiCo alloy Schottky contacts on ntype molecular-beam epitaxy GaAs," Journal of Applied Physics, vol. 91, no. 1, pp. 245-250, 2002.

[47] N. Tuğluoğlu, S. Karadeniz, M. Şahin, and H. Şafak, “Temperature dependence of current-voltage characteristics of $\mathrm{Ag} / \mathrm{p}$ SnSe Schottky diodes," Applied Surface Science, vol. 233, no. 1-4, pp. 320-327, 2004. 

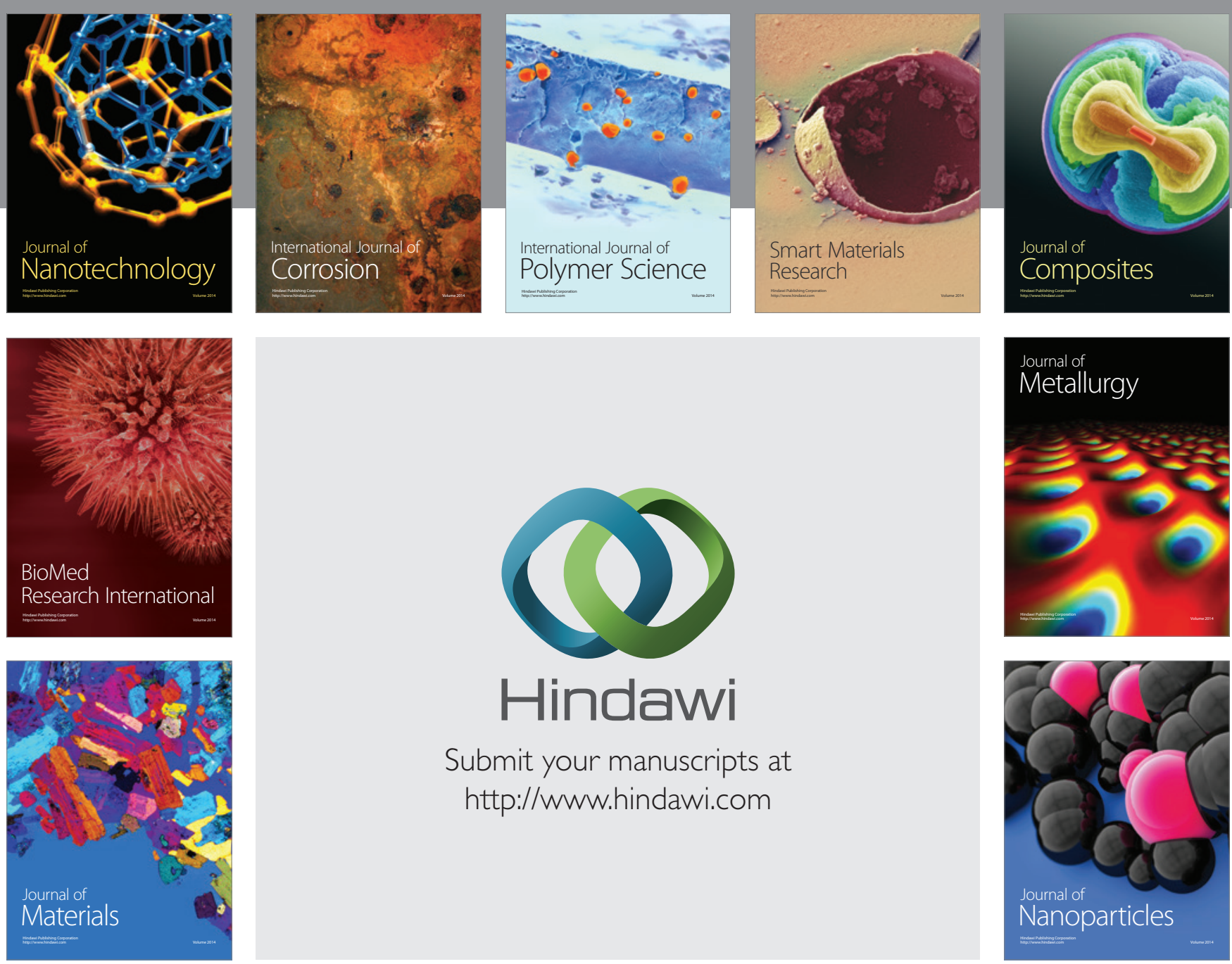

Submit your manuscripts at http://www.hindawi.com
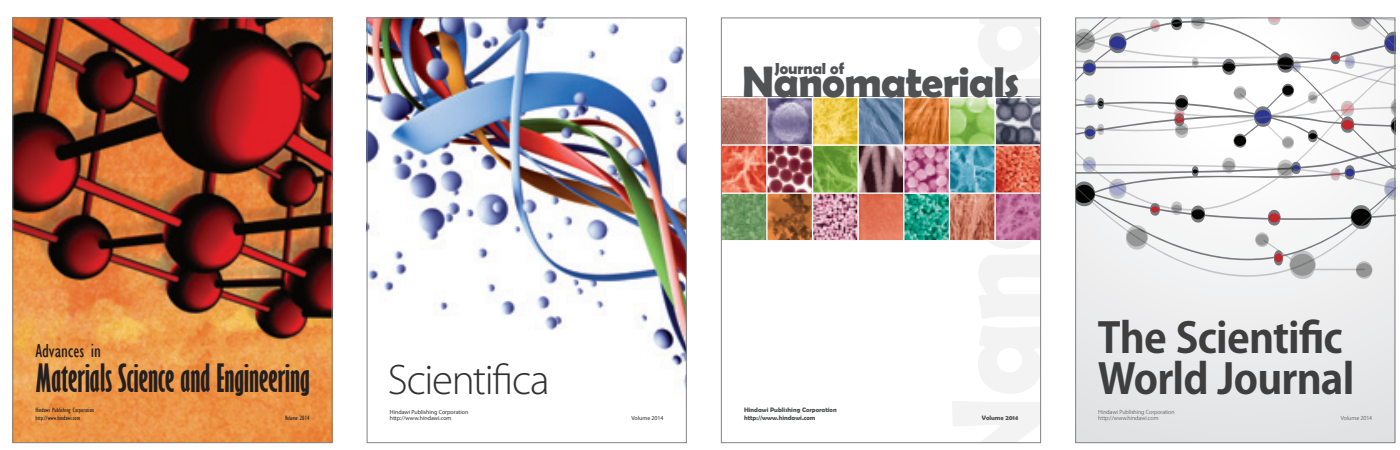

\section{The Scientific World Journal}
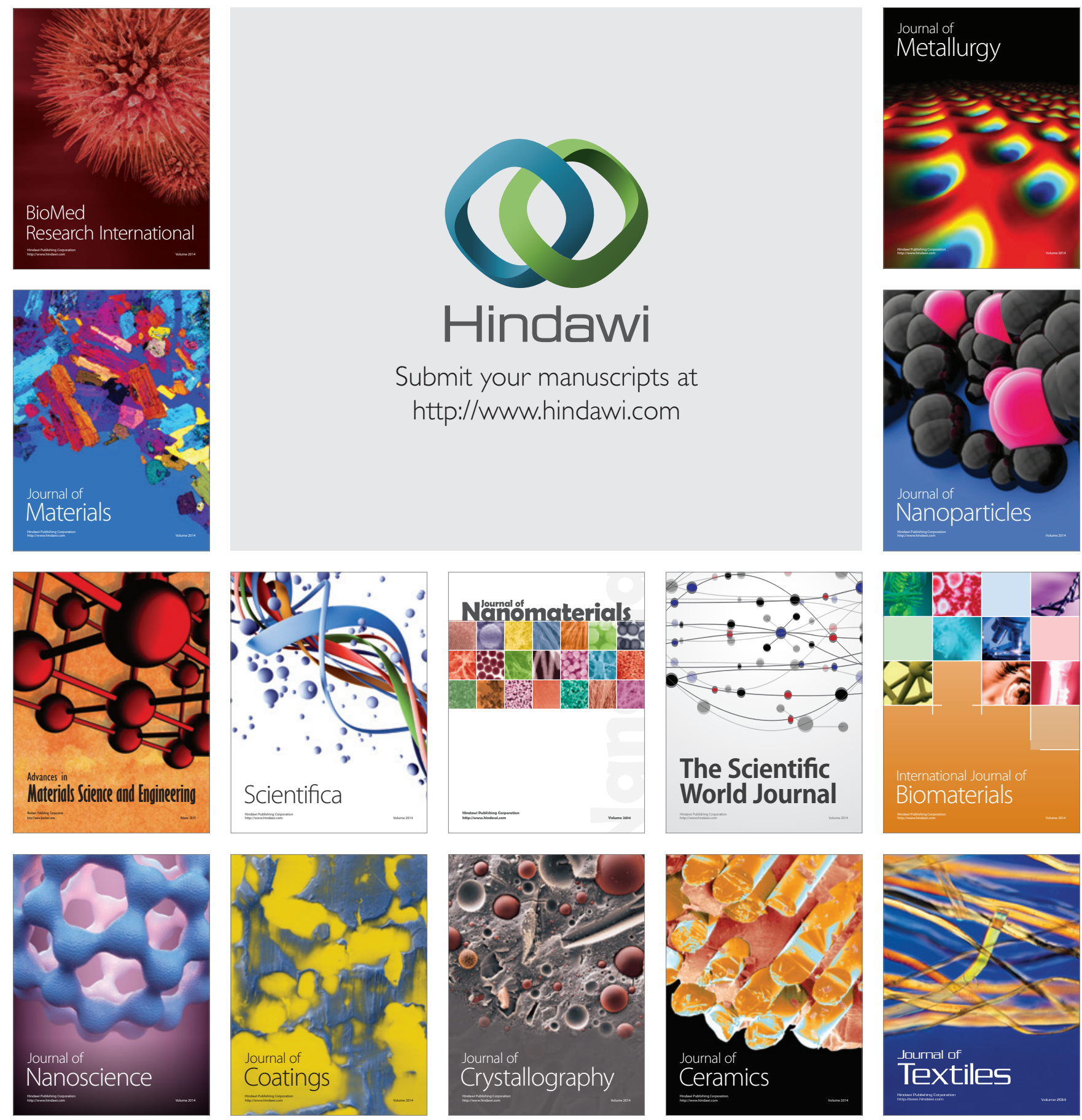